\title{
High-power and frequency-stable ultraviolet laser performance in space for the wind lidar on Aeolus
}

\author{
Oliver lux, ${ }^{1, *}$ Denny Wernham, ${ }^{2, *}$ Paolo Bravetti, ${ }^{3}$ Phil McGoldrick, ${ }^{4}$ \\ Olivier leCrenier, ${ }^{5}$ Wolfgang Riede, ${ }^{6}$ Alessandro D'OttaVi, ${ }^{7}$ Valeria De \\ SANCTIS, ${ }^{7}$ MARC SCHILlinger, ${ }^{5}$ Jérémie LOCHARD, ${ }^{5}$ JON MARSHALL, ${ }^{4}$ CHRISTIAN \\ LeMmerz, ${ }^{1}$ Fabian WeILER, ${ }^{1}$ LINDA Mondin, ${ }^{2}$ Alessandra CIAPPONI, ${ }^{2}$ ThOMAS \\ Kanitz, ${ }^{2}$ Anders ElfVing, ${ }^{2}$ Tommaso Parrinello, ${ }^{8}$ ANd Oliver ReitebuCH ${ }^{1}$
}

\author{
${ }^{1}$ German Aerospace Center (Deutsches Zentrum für Luft- und Raumfahrt), Institute of Atmospheric Physics, 82234 Oberpfaffenhofen, Germany \\ ${ }^{2}$ European Space Agency-ESTEC, Keplerlaan 1, Noordwijk, NL-2201AZ, The Netherlands \\ ${ }^{3}$ Airbus Italia S.p.A., Via dei Luxardo, 22-24, 00156 Rome, Italy \\ ${ }^{4}$ Airbus Defence and Space (Stevenage), Gunnels Wood Rd, Stevenage SG1 2AS, United Kingdom \\ ${ }^{5}$ Airbus Defence and Space (Toulouse), Rue des Cosmonautes, 31400 Toulouse, France \\ ${ }^{6}$ German Aerospace Center (Deutsches Zentrum für Luft- und Raumfahrt), Institute of Technical Physics, 70569 Stuttgart, Germany \\ ${ }^{7}$ Leonardo S.p.A., Via Industria, 4, 00040 Pomezia RM, Italy \\ ${ }^{8}$ European Space Agency-ESRIN, Largo Galileo Galilei, 1, 00044 Frascati RM, Italy \\ *Corresponding authors: Oliver.Lux@dlr.de, Denny.Wernham@esa.int
}

Received XX Month XXXX; revised XX Month, XXXX; accepted XX Month XXXX; posted XX Month XXXX (Doc. ID XXXXX); published XX Month XXXX

\begin{abstract}
Global acquisition of atmospheric wind profiles using a spaceborne direct-detection Doppler wind lidar is being accomplished since the launch of ESA's Aeolus mission. One key part of the instrument is a single-frequency, ultraviolet laser which emits nanosecond laser pulses into the atmosphere. High output energy and frequency stability ensure sufficient signal-to-noise ratio of the backscatter return and accurate determination of the Doppler frequency shift induced by the wind. This paper discusses the design of the laser transmitter for the first Doppler wind lidar in space and its performance during the first year of the Aeolus mission, providing valuable insights for upcoming lidar space missions. (C) 2020 Optical Society of America
\end{abstract}

http://dx.doi.org/10.1364/OL.99.099999

On 22nd August 2018, the European Space Agency (ESA) launched its fifth Earth Explorer mission - Aeolus, which is named after the Greek god and "keeper of the winds". The launch marked an important milestone in the centennial history of atmospheric observing systems, as Aeolus is the first mission to directly acquire atmospheric wind profiles from the ground to the lower stratosphere on a global scale [1-3]. For this purpose, Aeolus carries the first-ever satellite-borne wind lidar ALADIN (Atmospheric LAser Doppler INstrument) as its single payload [4]. Whilst orbiting the Earth on a Sun-synchronous orbit at $320 \mathrm{~km}$ altitude with a repeat cycle of seven days, ALADIN measures one component of the wind vector along the instrument's line-of-sight (LOS) with a vertical resolution of $0.25 \mathrm{~km}$ to $2 \mathrm{~km}$ depending on altitude. The major objective of the Aeolus mission is the assimilation of wind observations in order to improve the accuracy of numerical weather prediction (NWP) models [5,6]. Particularly, wind profiles acquired over the oceans the tropics, and in the Southern Hemisphere contribute to closing large gaps in the global wind data coverage which represented a major deficiency in the Global Observing System before the launch of Aeolus [7].

Over the past decades, diverse types of spaceborne lidars have been developed for gaining information on various atmospheric parameters at a global scale [8]. In 1994, the pioneering Lidar In-Space Technology Experiment (LITE) on the Space Shuttle Discovery was the first to use a spaceborne lidar for atmospheric studies [9]. The space lidar mission ICESat (Ice, Cloud and land Elevation Satellite) was in operation from 2003 through 2009 incorporating the Geoscience Laser Altimeter System (GLAS) which measured surface elevation, the height of dense clouds and the vertical distribution of aerosol and thinner clouds [10]. Since the end of 2018, the ICESat- 2 mission operates the Advanced Topographic Laser Altimeter System (ATLAS) for measurements of sea ice freeboard and ice sheet elevation. Another breakthrough was the launch of CALIPSO (Cloud-Aerosol Lidar and Infrared Pathfinder Satellite Observation) in 2006, carrying CALIOP (Cloud-Aerosol Lidar with Orthogonal Polarization) - the first lidar for aerosol and cloud observations from space which is still in operation [11]. Between 2015 and 2018, the Cloud-Aerosol Transport System (CATS) onboard the International Space Station (ISS) enabled the acquisition of cloud and aerosol profiles [12]. Apart from LITE which included an ultraviolet (UV) laser operable for only two weeks and CATS, the transmitters integrated in the aforementioned spaceborne lidar instruments are based on lasers emitting in the visible and infrared (IR) spectral region. They are characterized by exceptional designs and remarkable system 
parameters [13]. However, in terms of its spectral properties and the durability of the optics exposed to high-energy UV pulses, the laser developed for the Aeolus mission is unprecedented.

ALADIN is composed of a pulsed, frequency-stabilized, UV laser transmitter, a 1.5-m diameter Cassegrain-type telescope in monostatic configuration (where the signal emission and reception are realized via the same telescope), and a dual-channel receiver sensitive for both molecular and particle backscatter from clouds and aerosols [4]. The wind measurement principle of ALADIN relies on detecting frequency differences between the emitted and the backscattered laser pulses. Due to the Doppler effect, the frequency $f_{0}$ of the outgoing pulse is shifted upon backscattering from particles and molecules which move with the ambient wind. The frequency shift in the backscattered signal $\Delta f_{\text {Doppler }}$ is proportional to the wind speed $v_{\text {Los }}$ along the laser beam LOS according to $\Delta f_{\text {Doppler }}=2 f_{0} v_{\mathrm{LOS}} / c$, where $c$ is the speed of light. Emitted and backscattered light are characterized through a reference and an atmospheric path. Given that a LOS wind speed of $1 \mathrm{~m} / \mathrm{s}$ translates to a frequency shift of $5.63 \mathrm{MHz}$ (at $f_{0}=844.75 \mathrm{THz}$ ), the required accuracy of the frequency measurement is on the order of $10^{-8}$ to measure wind speeds with an accuracy of $1 \mathrm{~m} / \mathrm{s}$. Due to the large difference in spectral width of the particulate Mie $(\sim 50 \mathrm{MHz})$ and molecular Rayleigh-Brillouin $\left(\sim 3.8 \mathrm{GHz}\right.$ at $f_{0}$ and atmospheric temperature of $293 \mathrm{~K}$ ) atmospheric backscatter signals, two complementary channels are used for deriving the Doppler frequency shift from the two spectral contributions separately. While the Mie channel is based on a Fizeau interferometer, two sequential Fabry-Pérot interferometers (FPIs) are employed for measuring the Doppler shift from the molecular return in the Rayleigh channel.

ALADIN is equipped with two fully redundant, switchable laser transmitters, referred to as flight models A and B. They are based on frequency-tripled, diode-pumped Nd:YAG laser systems emitting at $354.89 \mathrm{~nm}$ and consist of five main stages [4,14,15], as shown in Fig. 1. The narrowband seed radiation from a nonplanar Nd:YAG ring laser (NPRO), which is frequency-locked to a second, cavity-locked NPRO, is fiber-coupled into the folded cavity of the $80-\mathrm{cm}$ long Q-switched master oscillator (MO) whose length is actively controlled with a piezo actuator for each laser pulse in order to find the optimal condition for single mode operation [15]. The output pulses from the MO $(50.5 \mathrm{~Hz}$ repetition rate) are amplified in two amplifiers, the first in double-pass and the second in single-pass, each being realized by side-pumped and conductively cooled Nd:YAG zigzag slabs. The amplified IR beam is then guided to the harmonic generation stage comprising a set of nonlinear lithium triborate (LBO) crystals to generate UV output pulses (20 ns FWHM [14]) with a conversion efficiency of about 30\%. Finally, the UV beam pointing is adjusted by a diasporameter to suit the ALADIN optics.

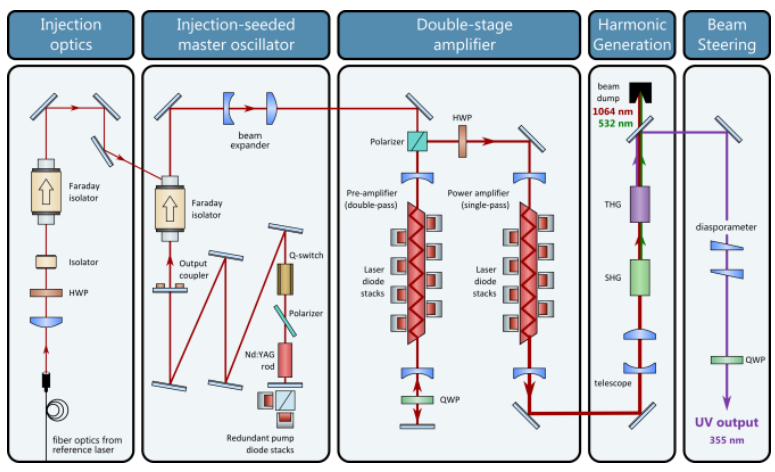

Fig. 1. ALADIN laser scheme. HWP: half-wave plate, QWP: quarterwave plate, SHG/THG: second/third harmonic generator.
Prior to their implementation in the ALADIN instrument, extensive test campaigns were performed on all critical laser optics to identify suitable high-power laser optics and reliable coating processes [16]. The extension of the ISO test standards on test methods for laserinduced damage (LID) threshold for larger test areas and long endurance tests, in addition to an increased operational fluence safety margin, were important steps for reliable operation of the ALADIN laser. The latter also requires an ultra-clean environment which is accomplished by a constant purge of the laser as well as the emission and reception optics with pure molecular oxygen gas at a pressure of $50 \mathrm{~Pa}$ and $100 \mathrm{~Pa}$, respectively. The oxygen environment prevents the build-up of laser-induced contamination (LIC) which, together with LID, were identified as the most limiting factors for the lifetime of the UV laser, and hence the Aeolus mission [17]. Replenishment of the oxygen environment is mandatory to ensure a low-level oxygen purge of the optics and is achieved by the so-called In-situ Cleaning System (ICS). The ICS is composed of two oxygen tanks, pressure regulators and valves to accurately control the pressures in the sealed cavities of the lasers and emission path optics up to the telescope. The oxygen supply is sufficiently large to ensure a lifetime of at least three years.

Before switching on the laser a few days after the launch of Aeolus, the ICS was used to prime the instrument sealed volumes twice in order to expunge any residual contamination. Afterwards, the ICS was set to operate the instrument at the operational oxygen pressure levels. The laser transmitter was then switched-on in successive discrete steps of increasing energy until the maximum energy set-point was reached on $4^{\text {th }}$ September 2018. Since then, ALADIN has been measuring vertical wind profiles at a global scale $[2,3]$.

Soon after the beginning of operation, a decreasing trend in the first flight model laser (FM-A) output energies, particularly the UV emit energy, was observed from internal photodiodes (PDs) placed at different stages of the laser transmitter. Aside from the fact that the initial UV energy of $65 \mathrm{~mJ}$ was lower than the expected level of $80 \mathrm{~mJ}$ which was reached on-ground, this parameter dropped by an average of $-0.4 \mathrm{~mJ}$ per day during the first 14 days after power-on, as depicted in Fig. 2. Consequently, several adjustments to the laser parameters were carried out to alleviate the energy loss. In mid-September 2018, thermal adjustments were made to the two amplifiers, leading to a better temperature distribution of the laser optical bench. As a result, the UV energy increased by $3 \mathrm{~mJ}$, while its decrease rate was considerably reduced to $-0.14 \mathrm{~mJ} /$ day, or $1 \mathrm{~mJ} /$ week correspondingly. This decay rate was comparable to that observed on-ground of the FMA laser. Several temporary adjustments of the amplifier currents (laser tests) were made in order to check the impact on the laser energy and fluence, and a permanent adjustment was made on $15^{\text {th }}$ December 2018, elevating the UV energy by $12 \%$ to $57 \mathrm{~mJ}$ from which it dropped by the same rate of $-1 \mathrm{~mJ} /$ week until the end of the four-month commission phase.

On $15^{\text {th }}$ January 2019, a reboot anomaly on the GPS unit of the satellite caused a timing synchronization issue with the instrument detection chain and triggered an ALADIN instrument switch-off. This incident resulted in an interruption of instrument operations for around one month before the laser was fully powered again. It is interesting to note that the energy degradation trend continued during the period when the laser was off (the amplifiers were operating during the majority of this non-lasing period), suggesting that the degradation was not due to the presence of the laser beam, i.e. not due to LID or LIC. The FM-A laser continued to be operated up to June 2019 when the energy had reached $40 \mathrm{~mJ}$ and it was decided to switch to the second flight laser (FM-B) to ensure a sufficient signal-to-noise ratio of the backscatter return, and hence, low random error of the wind observations. The FM-B was switched-on using the same procedures as for the first laser and reached an initial energy of $67 \mathrm{~mJ}$. 


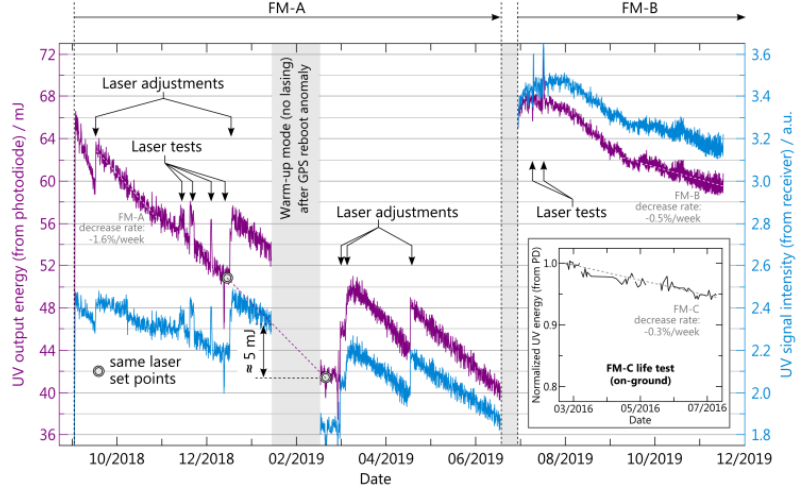

Fig. 2. Temporal evolution of the UV energy during nominal wind mode measured with an internal photodiode (purple) and of the receiver reference signal intensity measured on the FPIs channel (blue). The inset shows the energy evolution of the ground-based FM-C laser during a life test in 2016.

A laser test in July 2019 demonstrated that the energy could be increased to $72 \mathrm{~mJ}$ when the laser radiator temperature was adjusted by $-0.2 \mathrm{~K}$. This energy level was close to that obtained in the ground thermal vacuum test, leading to the conclusion that any of the degradation observed on the first laser had not impacted the second laser. In addition to the expected losses induced by the ageing of the laser diodes, further investigations revealed that a large part of the energy degradation of the FM-A was most likely due to a progressive misalignment of the $\mathrm{MO}$, resulting in a reduction of MO energy and consequently UV energy. This needs to be confirmed with a dedicated MO lasing threshold test, but, if correct, means that the FM-A can be eventually recovered by increasing the $\mathrm{MO}$ energy later in the mission.

In addition to the energy monitoring by means of the internal PD, the temporal evolution of the UV emit energy was also assessed from the signal intensity measured in the reference path of the instrument's receiver. In particular, the small portion of the output radiation which is directed to the FPIs of the Rayleigh channel for determining the frequency of the emitted pulses $f_{0}$, was tracked over the course of the mission (blue curve in Fig. 2) and revealed a considerably lower relative decrease rate after the first laser adjustment $(-0.9 \% /$ week compared to $-1.6 \%$ /week measured with the photodiode), so that the signal intensity even exceeded the initial value after the adjustment on $15^{\text {th }}$ December 2018. The reason for this discrepancy has not been unambiguously identified, yet. As there are apertures between the PD and the instrument reference path, it could be due to the change in laser beam divergence or alignment drifts in the laser caused by the increase of the amplifier currents, or a change in the calibration curve of the UV PD Since the receiver signal level is also important for the wind retrieval, it is considered the more relevant source of information regarding the laser energy evolution.

The FM-B laser energy trend is markedly different to that of FM-A and appears to be more affected by seasonal temperature variations that influence the satellite platform. After an initial increase due to thermalisation, the UV energy was quite stable for about one month. This was followed by a drop in energy over a two-month period and a stabilization to a decrease rate of about $-0.3 \mathrm{~mJ} /$ week $(-0.5 \%$ /week), which is expectable from laser diode ageing. A comparable rate was measured for FM-B on-ground, while even lower rates were observed for a third laser model (FM-C) during a life test in 2016 (see inset of Fig. 2). It should be pointed out that no adjustments have been made to FM-B in the five months after switch-on, and, unlike FM-A, there are significant margins for energy adjustment via phasing of the amplifiers with the Q-switched pulse, giving additional confidence that the instrument will meet its three-year mission lifetime requirement.
An independent measurement of the laser absolute frequency and its temporal stability, e.g. by using a wavemeter as done during on-ground tests [18], is not available in space. However, the spectrometer data gained from the Fizeau interferometer of the Mie channel represents an accurate source of information and is thus used for determining the spectral properties of the narrowband laser emission, shot-to-shot, from the reference signal. Conversion of the Mie response variations into relative laser frequency variations is based on dedicated in-flight calibrations. The accuracy of the "inherent wavemeter" is about $1 \mathrm{MHz}$ (UV) and mainly limited by shot-noise-limited signal-to-noise ratio of the Mie signal and the width of the Fizeau interference fringe whose centroid positon relates almost linearly with the Doppler shift.

A typical time series of the laser frequency over $100 \mathrm{~min}$ (a bit more than one orbit) measured on $16^{\text {th }}$ September 2018 with FM-A is depicted in Fig. 3(a). A selected five-minute period from the beginning of the measurement (Fig. 3(b)) shows very low fluctuations of $4.9 \mathrm{MHz}$ (rms) which is comparable to the frequency stability of the ALADIN Airborne Demonstrator featuring a very similar laser design [19]. However, the time series also exhibits numerous sporadic periods where higher frequency variations occur along the orbit. During these periods, the fluctuations increase to more than $20 \mathrm{MHz}$ (rms) with peak-to-peak variations of up to $150 \mathrm{MHz}$ (Fig. 3(c)). A possible correlation to vibrations introduced by the satellite platform which affects the MO cavity length and, in turn, the laser frequency on time scales of a few seconds has not been found, yet. Despite the periods of increased jitter, the frequency stability for the scene in Fig.3(a) is $7.0 \mathrm{MHz}$ (rms). The overall pulse-to-pulse stability is in line with the requirements, despite the periods of enhanced frequency jitter. This frequency stability performance, which is evident for both FM-A and FM-B, is unrivalled by any high-power, spaceborne laser so far.

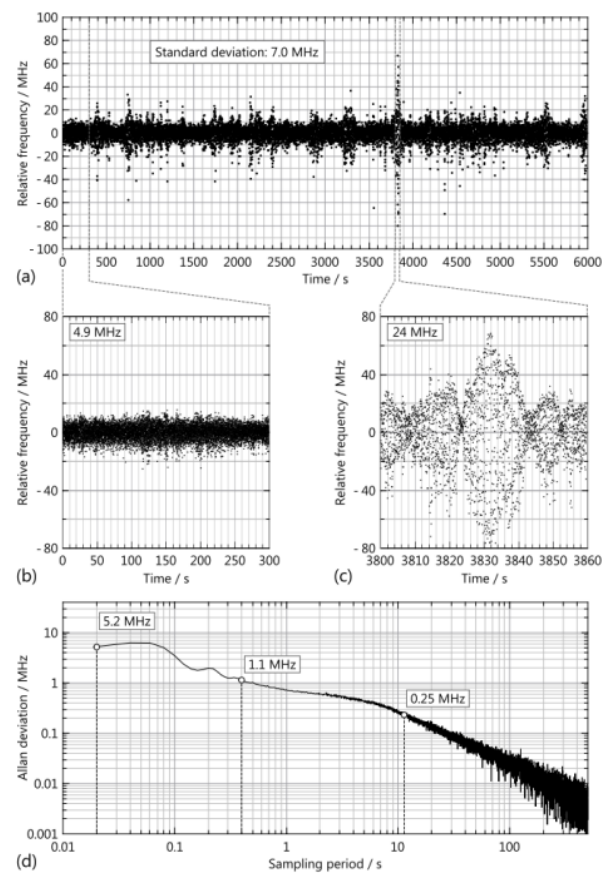

Fig. 3. Frequency stability of the ALADIN laser: (a) time series of the laser frequency fluctuations over one orbit of the Aeolus satellite measured with the Fizeau interferometer of the Mie channel; the middle panels show selected periods with very high stability (b) and enhanced frequency jitter (c). The bottom plot (d) represents the Allan deviation calculated for the time series in panel (a). The vertical dashed lines indicate sampling periods relevant for the wind retrieval. 
Note that $1 \mathrm{MHz}$ frequency stability of the master oscillator, corresponding to $3 \mathrm{MHz}$ of the frequency-tripled output, requires a cavity length stabilization of $3 \mathrm{~nm}$ within one oscillator free spectral range of $532 \mathrm{~nm}$ or $180 \mathrm{MHz}$, considering the $0.8 \mathrm{~m}$ resonator length. The Allan deviation plot in Fig. 3(d) provides further information on the frequency stability on time scales that are relevant for the wind retrieval. The latter is performed after accumulation of the signals from either 20 pulses on-board (so-called measurement level) or 600 laser pulses on-ground (observation level), corresponding to sampling periods of $0.4 \mathrm{~s}$ and $12 \mathrm{~s}$, respectively. The frequency stability is 1.1 $\mathrm{MHz}$ and $0.25 \mathrm{MHz}$ which translates to a wind random error introduced by laser frequency fluctuations of as low as $0.2 \mathrm{~m} / \mathrm{s}$ and $0.04 \mathrm{~m} / \mathrm{s}$. The high frequency stability of the laser on longer time scales ( $>12 \mathrm{~s}$ ) reduces the necessity to perform regular response calibrations of the receiver spectrometers for ensuring low systematic wind errors.

The launch of Aeolus on 22 August 2018 was a seminal event in the history of spaceborne active remote sensing, since, after more than two decades of development and testing, one of the most ambitious lidars was finally put into orbit. Since its start of operation, ALADIN has been delivering global wind profiles for improving weather forecasts and studying atmospheric dynamics. A first assessment of the impact of the Aeolus data on NWP shows positive results, particularly in the tropics and southern hemisphere [6].

At the heart of ALADIN is a unique laser transmitter fulfilling a set of strict requirements that have not been simultaneously met by any existing laser so far. In particular, it generates powerful, singlefrequency UV laser pulses at high efficiency in a compact and lightweight conductively cooled package. Over the course of the fourmonth commissioning phase, the output energy of the first flight laser was observed to decrease by about $1 \%$ per week which is primarily traced back to an alignment drift of the master oscillator. In summer of 2019, a switch-over to the redundant laser aboard Aeolus was successfully performed to ensure a sufficiently low random error of the wind data. In accordance with on-ground tests, the second laser showed better performance producing around $65 \mathrm{~mJ}$ of pulse energy at a significantly lower decrease rate of about $-0.5 \%$ per week during the first five months of operation. Nevertheless, a switch back to the first laser and execution of an energy recovery procedure are kept as an option. By the end of 2019, the two ALADIN lasers have accumulated around 1.9 billion high-energy laser shots in 15 months of operations. The better performance of the second laser along with the additional margins for increasing the energy on this unit, gives good confidence that Aeolus can meet its mission lifetime of three years, indeed. Currently, the life time is most likely limited by the number of cycles of the latch valves on the oxygen supply system, which have only been qualified for just over the number required to complete the three year mission, or an unexpected degradation in the laser transmitter.

Established performance verification methods based on receiver data are being used for monitoring the laser energy, beam profile and alignment as well as the frequency stability. The latter is characterized by exploiting the high precision of one of the two receiver spectrometers, revealing a pulse-to-pulse fluctuation of about $5 \mathrm{MHz}$ and as low as $250 \mathrm{kHz}$ on the time scale (12 s) of one wind observation (averaging along-track over $86 \mathrm{~km}$ ). Periods of enhanced frequency jitter with peak-to-peak variations of up to $150 \mathrm{MHz}$ are evident and subject of current studies. With regards to the development of novel laser sources for future lidar applications in general [20-22], and particularly the upcoming space missions EarthCARE (Earth Clouds, Aerosols and Radiation Explorer) [23], ACDL (Aerosol \& Carbon Detection Lidar) [24] and MERLIN (Methane Remote Sensing Lidar Mission) [25] which are scheduled for launch within the next five years, Aeolus is expected to yield valuable insights to the behavior of frequency-stabilized and high-energy lasers operating in space.
Funding. European Space Agency (ESA, 4200016312/02/NL/MM, 4000126336/18/I-BG)

Acknowledgment. The authors greatly acknowledge Anne Grete Straume and the Aeolus Mission Advisory Group, the Aeolus Space and Ground Segment Operations teams, the Aeolus Data Science and Innovation Cluster (DISC), the European Laser Risk Reduction Working Group and the Technical Directorate of the European Space Research and Technology Centre for their invaluable contributions.

Disclosures. The authors declare no conflicts of interest.

\section{REFERENCES}

1. J. L. Stith, D. Baumgardner, J. Haggerty, R. M. Hardesty, W.-C. Lee, D. Lenschow, P. Pilewskie, P. L. Smith, M. Steiner, and H. Vömel, Meteor. Monogr. 59, 2.1 (2018).

2. T. Kanitz, J. Lochard, J. Marshall, P. McGoldrick, O. Lecrenier, P. Bravetti, O. Reitebuch, M. Rennie, D. Wernham, and A. Elfving, Proc. SPIE 11180, 111801R (2019).

3. O. Reitebuch et al., Proc. 29th International Laser-Radar Conference (24.06.2019-28.06.2019).

4. ESA, ADM-Aeolus Science Report, ESA SP-1311, E. Andersson and P. Clissold (eds.) (ESA Communications Production Office, 2008).

5. D. G. H. Tan and E. Andersson, Q. J. R. Meteorol. Soc. 131, 1737 (2005).

6. M. Rennie and L. Isaksen, Living Planet Symposium, (ESA, 13.05.2019 17.05.2019).

7. W. E. Baker et al., Bull. Amer. Meteor. Soc. 95, 543(2014).

8. F. Fouladinejad, A. Matkan, M. Hajeb, and F. Brakhasi, Int. Arch. Photogramm. Remote Sens. Spatial Inf. Sci. XLII-4/W18, 407 (2019).

9. D. M. Winker, R. H. Couch, and M. P. McCormick, Proc. IEEE 84, 164 (1996).

10. J. B. Abshire, X. Sun, H. Riris, J. M. Sirota, J. F. McGarry, S. Palm, D. Yi, and P. Liiva, Geophys. Res. Lett. 32, L21S02 (2005).

11. D. Winker, M. Vaughan, and B. Hunt, Proc. SPIE 6409, 640902 (2006).

12. M. J. McGill, J. E. Yorks, V. S. Scott, A. W. Kupchock, and P. A. Selmer, Proc. SPIE 9612, 96120A (2015).

13. F. Hovis, N. Sawruk, T. Chuang, and R. Burnham, Advanced Solid-State Lasers Congress (OSA), LW2B.1 (2013).

14. A. Cosentino, A. D'Ottavi, A. Sapia, and E. Suetta, 2012 IEEE International Geoscience and Remote Sensing Symposium (IEEE, 22.07.2012 27.07.2012), pp. 5673-5676.

15. A. Cosentino, A. Mondello, A. Sapia, A. D'Ottavi, M. Brotini, E. Nava, E. Stucchi, F. Trespidi, C. Mariottini, P. Wazen, N. Falletto, and M. Fruit, Proc. SPIE 10568, 1056817 (2017).

16. W. Riede, P. Allenspacher, A. Ciapponi, H. Schröder, D. Wernham, F. Era, and D. Thibault, Proc. SPIE 10564, 105642K (2018).

17. L. Mondin, F. F. Era, H. Kheyrandish, A. Bolkhovitinov, A. Baselga Mateo, M. Hippler, D. Thibault, H. Schröder, D. Wernham, A. Ciapponi, C. Heese, T. Ivanov, G. van Papendrecht, W. Riede, and Y. Butenko, Proc. SPIE 10562, 105621U (2017).

18. L. Mondin and P. Bravetti, Proc. SPIE 10563, 105633B (2017).

19. C. Lemmerz, O. Lux, O. Reitebuch, B. Witschas, and C. Wührer, Appl. Opt. 56, 9057 (2017).

20. M. Strotkamp, F. Elsen, J. Löhring, M. Traub, and D. Hoffmann, Appl. Opt. 56, 2886 (2017).

21. S. Hahn, M. Bode, J. Luttmann, and D. Hoffmann, Proc. SPIE 10563, 105630Q (2017).

22. F. Hovis, M. Albert, F. Fitzpatrick, K. Puffenburger, T. Schum, D. J. J. Rudd, and S. Litvinovitch, 2017 IEEE International Geoscience and Remote Sensing Symposium (IEEE, 23.07.2017 - 28.07.2017), pp. 4216-4219.

23. A. J. Illingworth et al., Bull. Amer. Meteor. Soc. 96, 1311 (2015).

24. D. Liu, Z. Zheng, W. Chen, Z. Wang, W. Li, J. Ke, Y. Zhang, S. Chen, C. Cheng, and S. Wang, Opt. Express 27, A481 (2019).

25. G. Ehret et al., Remote Sens. 9, 1052 (2017). 